\title{
Validation of a novel device to objectively measure adherence to long-term oxygen therapy
}

\author{
Sun-Kai V Lin' \\ Daniel K Bogen' \\ Samuel T Kuna ${ }^{2,3}$ \\ 'Department of Bioengineering; \\ ${ }^{2}$ Department of Medicine, Pulmonary, \\ Allergy and Critical Care Division, \\ and Center for Sleep and Respiratory \\ Neurobiology, University \\ of Pennsylvania, Pennsylvania, USA; \\ ${ }^{3}$ Department of Medicine, Philadelphia \\ Veterans Affairs Medical Center \\ Philadelphia, Pennsylvania, USA
}

Rationale: We have developed a novel oxygen adherence monitor that objectively measures patient use of long-term oxygen therapy. The monitor attaches to the oxygen source and detects whether or not the patient is wearing the nasal cannula.

Objective: The study's purpose was to validate the monitor's performance in patients with chronic obstructive pulmonary disease during wakefulness and sleep.

Methods: Ten adult males with stable chronic obstructive pulmonary disease (mean \pm SD $\mathrm{FEV}_{1} 37.7 \pm 14.9 \%$ of predicted) on long-term continuous oxygen therapy were tested in a sleep laboratory over a 12-13 hour period that included an overnight polysomnogram.

Measurements: The monitor's measurements were obtained at 4-minute intervals and compared to actual oxygen use determined by review of time-synchronized video recordings.

Main results: The monitor made 1504/1888 (79.7\%) correct detections (unprocessed data) across all participants: 957/1,118 (85.6\%) correct detections during wakefulness and 546/770 (70.9\%) during sleep. All errors were false negatives, ie, the monitor failed to detect that the participant was actually wearing the cannula. Application of a majority-vote filter to the raw data improved overall detection accuracy to $84.9 \%$.

Conclusions: The results demonstrate the monitor's ability to objectively measure whether or not men with chronic obstructive pulmonary disease are receiving their oxygen treatment. The ability to objectively measure oxygen delivery, rather than oxygen expended, may help improve the management of patients on long-term oxygen therapy.

Keywords: chronic obstructive pulmonary disease

Long-term oxygen therapy (LTOT) is a well-established treatment for patients with oxygen desaturation due to parenchymal lung diseases (NOT 1980; MRC 1981). In patients with chronic obstructive pulmonary disease (COPD) and hypoxemia at rest, continuous use of LTOT ( $>15$ hours/day) improves survival and quality of life, and reduces the number of hospitalizations (Criner 2000; Pierson 2000). Unfortunately, this therapy is costly, accounting for approximately $30 \%$ (US $\$ 1.3$ billion) of annual Medicare expenditures for durable medical equipment (Dunne 2000). It is likely that many of these dollars are wasted because studies report that many patients do not adhere adequately to this treatment (Howard et al 1992; Granados et al 1997; Avdeev et al 1999).

Compounding this problem, current methods used to measure adherence to LTOT probably overestimate actual use. Each type of oxygen source used for LTOT requires a different method to objectively assess patient adherence to treatment. For the oxygen concentrator, average daily use is estimated by recording the concentrator's hour-meter readings ("power on" time) over a known period of time. For oxygen tanks, adherence is estimated by knowing the prescribed flow rate and the number of tanks used. For liquid oxygen, adherence is calculated by weighing the container, knowing the flow rate, and 
estimating the amount of evaporation and venting from the system. All of the above measures are deficient and probably misleading because: 1) they do not discern whether or not the patient is actually inhaling the oxygen, as opposed to merely having the oxygen source turned on, ie, oxygen expenditure may not reflect oxygen delivery to the patient, and 2) they fail to provide information regarding the timing of oxygen use within the day. The lack of an accurate, objective measure of adherence to LTOT prevents the accurate determination of the optimal duration of daily treatment. In addition, the inability to identify patients with inadequate adherence prevents the development of interventions designed to improve adherence and thereby to increase the effectiveness of this expensive but life-saving treatment.

We have developed a novel oxygen adherence monitor to address the above limitations and objectively document when a patient is actually receiving oxygen treatment (Lin et al 2006). The monitor attaches to the oxygen source and detects pressure in the tubing, including the respiratory-related pressure fluctuations transmitted from the nares. The monitor is designed to detect when the oxygen source is turned on and when the patient is actually wearing the nasal cannula and receiving treatment. Our monitor performs measurements every four minutes over a 25 -second duration and, for each measurement, identifies one of four conditions: source-on/ cannula-on, source-on/cannula-off, source-off/cannula-off, and source-off/cannula-on. Periodic measurement, as opposed to continuous measurement, was selected to extend battery life of the AA battery powered monitor. The design and operating characteristics of the device have been previously detailed and the device has been validated in patients with COPD under controlled conditions during relatively short periods while sitting at rest and walking (Lin et al 2006). The purpose of the current study was to evaluate the monitor's performance in patients with COPD during wakefulness and sleep over a longer period of time and under less controlled conditions. Some of the results of these studies have been previously reported in the form of an abstract (Lin et al 2005).

\section{Methods}

\section{Participant selection}

Ten male adults with COPD using continuous LTOT via nasal cannula were enrolled (Table 1). The participants were recruited from veterans receiving care at the Philadelphia Veterans Affairs Medical Center, where men constitute about $85 \%$ of the patient population.

COPD was identified as a forced expiratory volume in one second $\left(\mathrm{FEV}_{1}\right) /$ forced vital capacity $(\mathrm{FVC})<70 \%$ and
Table I Clinical characteristics of the 10 participants

\begin{tabular}{ll}
\hline Measure & Mean \pm SD \\
\hline Age $(\mathrm{yr})$ & $64 \pm 7$ \\
Height $(\mathrm{cm})$ & $174.8 \pm 4.3$ \\
Weight $(\mathrm{kg})$ & $94.3 \pm 16.8$ \\
$\mathrm{FEV}_{\text {, }}(\mathrm{L})$ & $1.19 \pm 0.43$ \\
$\mathrm{FEV}_{\text {, }}(\%$ predicted) & $37.7 \pm 14.9$ \\
FVC (L) & $2.43 \pm 0.73$ \\
FEV /FVC (\%) & $59.6 \pm 16.3$ \\
\hline
\end{tabular}

Abbreviations: $\mathrm{FEV}_{1}$, forced expiratory volume in one second; FVC, forced vital capacity.

an $\mathrm{FEV}_{1}<80 \%$ of predicted without significant improvement following bronchodilator administration. None of the participants had a previous diagnosis of sleep apnea or other sleep disorder. The study was approved by the Institutional Review Boards at the Philadelphia VAMC and the University of Pennsylvania. Written informed consent was obtained from all participants.

\section{Oxygen adherence monitor}

The oxygen adherence monitor $(18 \times 10 \times 2 \mathrm{~cm})$ was attached by a " $\mathrm{T}$ " connection to the oxygen tubing near the oxygen source (Lin et al 2006). The monitor consists of a pneumatic filtering system and an electronic detection system. The pneumatic filtering system isolates the fluctuations in nasal pressure from the oxygen supply pressure. The electronic system consists of two pressure transducers, a microprocessor used to sample the data coming from the pressure transducers, a memory storage chip, and a serial port to allow cable connection for downloading data to a personal computer. The microprocessor is programmed to identify four different conditions (source-on/cannula-on, source-on/cannula-off, source-off/cannula-off, and source-off/cannula-on), record the exact time and date when each condition occurred, and tabulate the total time spent in each condition.

\section{Protocol}

The participants arrived at the sleep center at 16:00 and were administered supplemental oxygen at their prescribed flow rate $(2-3 \mathrm{~L} / \mathrm{min})$ through a nasal cannula with a $50-\mathrm{ft}$ length of tubing attached to the wall oxygen supply in their bedroom. Oxygen flow rate remained constant throughout the protocol. Therefore, the protocol tested two conditions: source-on/cannula-off and source-on/cannula-on.

The participants were aware of the purpose of the study and were instructed to remove the nasal cannula whenever 
they wished during wakefulness. Each participant performed an overnight polysomnogram while wearing the oxygen cannula. Prior to the start of the polysomnogram, they were encouraged to watch TV, read magazines, and walk in their bedroom. They were provided dinner between 18:00-19:00 and then changed into their bedtime clothes. Between 20:00-21:00, the sleep technologist attached the polysomnographic sensors while the participants sat in a chair. The participants went to bed at their regular bedtimes, and the polysomnograms were recorded using standard techniques (AASM 1999).

Two wide-angle infrared cameras were placed in the bedroom to determine whether or not the patient was wearing the oxygen cannula. Each camera was placed in an opposite corner of the room to obtain the largest viewable area. The video images from each camera were fed into a single surveillance VCR (Lorex Technology Inc., Markham, Ontario, Canada), that multiplexed the images from both cameras and time-stamped the frames. White-colored paper tags $(1 \times 2$ inches $)$ were placed on the nasal cannula tubing at each cheek so that the cannula was clearly visible on the video recording. Logs of the participants' activities were kept by the sleep technologists and were used to help interpret the images on the videotape.

Throughout the protocol, the subjects wore an ultra-thin nasal cannula with 4-foot length tubing securely taped to the oxygen cannula to obtain simultaneous, continuous nasal pressure recordings. During wakefulness, nasal pressure was recorded using a light-weight, battery powered pressure recorder (Stardust II, Respironics, Pittsburgh, PA, USA) that hung around the neck. During the polysomnogram, the ultra-thin nasal cannula was connected to a pressure transducer (Pro-Tech, Woodinville, WA, USA) with output to the polysomnographic computer.

All time-sensitive devices used in the study were timesynched to the oxygen adherence monitor at the beginning of the protocol.

\section{Data analysis}

The data from the oxygen adherence monitor were downloaded in text format for analysis. The polysomnograms were manually scored with the aid of computer software by a certified polysomnographic technologist using standard guidelines (ATS 1989; AASM 1999). All abnormal respiratory events were at least 10 seconds in duration. Apneas were defined by an absence of airflow as evidenced by the nasal pressure cannula signal. Hypopneas were defined as a greater than $50 \%$ reduction in a respiratory signal from baseline, or a less than $50 \%$ reduction in a respiratory signal from baseline that was associated with an arousal or an oxygen desaturation of at least 3\% (AASM 1999).

The monitor's measurement (cannula “on" vs. cannula "off") from each sampling period was compared to the same period of time on the videotape recording. Thirty out of the total 1,918 measurements $(<2 \%)$ occurred when the participants were out of camera view and were excluded. The accuracy of the monitor was assessed by computing the percentage of the monitor's measurements that were correct relative to the videotape. The simultaneous, continuous nasal pressure recordings were reviewed to evaluate possible explanations for any discrepancies between videotape and monitor, eg, the videotape showing that the cannula was "on", but the monitor detecting the event as cannula "off".

Recognizing that the overall error contribution during wakefulness and sleep was due to random false negatives, a post-processing majority-vote filter was applied to the data in an attempt to improve the accuracy of the monitor. The majority-vote filter, sometimes referred to as a majority logical filter, is a standard digital analysis technique commonly used to reduce noise in graphical data (Ting and Prasada 1980). The technique involves taking an odd-number of contiguous sample points, each of which is designated as a " 1 " or a "0", counting up which value is more frequent (ie, are there more " 1 's" or more " 0 's"?), and then assigning the more frequent value to the center point of the collection. Thus, the center point becomes a " 1 " if a majority of the data points are " 1 's." This algorithm is then applied to all possible same-size sample point collections in the data set.

In the present application, the majority-vote filter was applied to each sequence of five successive sample points with the "cannula-on" state designated as "1", and the "cannula-off" state as " 0 ". Thus, one can imagine the filter "moving along" the time sequence of data points, taking five at a time, determining the majority for those five, and then shifting along one time unit to process the next (overlapping) group of five points. If the majority of measurements $(\geq 3)$ within a particular sequence indicated that the participant was wearing the cannula, then the decision algorithm stipulated that the cannula was "on". If the majority of measurements indicated that the patient was not wearing the cannula, then the decision algorithm stipulated that the cannula was "off".

\section{Results}

Each participant was monitored for at least $12 \mathrm{hrs}$ (mean \pm $\mathrm{SD}=12.9 \pm 0.6)$. Time during wakefulness prior to the start of the polysomnogram ranged from 3 to $6 \mathrm{hrs}(5.0 \pm 0.8)$ and 
polysomnogram duration ranged from 6 to $9 \mathrm{hrs}(7.9 \pm 0.8)$. The polysomnographic results are presented in Table 2 .

The mean total sleep time was $5.4 \pm 1.8 \mathrm{hrs}$ and the mean sleep efficiency (total sleep time/recording time) was $68.3 \% \pm 23.5 \%$. The mean apnea-hypopnea index was $18.5 \pm 19.2$ events/hr (range 2.0 to 63.1 ). All 8 patients with an apnea-hypopnea index above 5 events/hr had obstructive sleep apnea, and there was no evidence of Cheyne-Stokes respiratory pattern. The apnea-hypopnea index was $<5$ events/hr in 2 participants, between 5 and 14.9 events/hr in 4 participants, between 15 and 29.9 events $/ \mathrm{hr}$ in 2 participants, and 30 events $/ \mathrm{hr}$ or greater in 2 participants. Minimum oxygen saturation was $86.4 \% \pm 10.5 \%$; and only $0.6 \% \pm 1.1 \%$ of the recording time was spent below $90 \%$ oxygen saturation.

Combining all data from the 10 participants, there were a total of 1,888 measurements (1,118 during wakefulness and 770 during sleep) (Table 3 ). The oxygen adherence monitor detected the correct condition in 1,504 measurements (unprocessed data), yielding an overall raw detection accuracy of $79.7 \%$. The monitor made 957/1,118 (85.6\%) correct detections during wakefulness and 546/770 (70.9\%) during sleep.

The monitor correctly detected all 47 measurements that occurred when the participants were not wearing the cannula, confirming the $100 \%$ specificity described in our previous validation study (Lin et al 2006). All errors (161 during wakefulness and 223 during sleep) were false negatives: ie, situations in which the monitor failed to detect that the participant was actually wearing the cannula. Prior to the polysomnogram recording, errors were more common during rest than during activities such as eating, talking, and dressing. Errors were more common during REM compared to NREM sleep. Overall, $73.3 \%$ of the

Table 2 Results of polysomnogram (PSG) recordings

\begin{tabular}{ll}
\hline Measure & Mean \pm SD \\
\hline Total time monitored before PSG $(\mathrm{hr})$ & $5.0 \pm 0.8$ \\
Total time monitored during PSG (hr) & $7.9 \pm 0.8$ \\
Total sleep time (hr) & $5.4 \pm 1.8$ \\
Time in NREM sleep (hr) & $4.5 \pm 1.8$ \\
Time in REM sleep (min) & $47.3 \pm 23.4$ \\
Sleep efficiency (\%) & $68.3 \pm 23.5$ \\
Apnea/hypopnea index (events/hr) & $18.5 \pm 19.2$ \\
Mean $\mathrm{O}_{2}$ saturation (\%) & $97.5 \pm 0.8$ \\
Minimum $\mathrm{O}_{2}$ saturation (\%) & $86.4 \pm 10.5$ \\
Time $\mathrm{O}_{2}$ saturation $<90 \%(\%)^{\mathrm{a}}$ & $0.6 \pm 1.1$ \\
\hline
\end{tabular}

Note: ${ }^{\text {a Percent }}$ of total sleep time that oxygen saturation was $<90 \%$. measurements were correct during NREM sleep whereas only $57.3 \%$ of the measurements obtained in REM sleep were correct.

Evaluating each individual's results, the mean percentage of accurate measurements over the entire recording period was $79.3 \pm 17.1 \%$. Results varied widely across participants, particularly during NREM and REM sleep (Table 3). Mean detection accuracy was $85.5 \pm 9.0 \%$ in wakefulness, $69.5 \% \pm 34.6 \%$ in NREM sleep, and $63.6 \pm 36.8 \%$ in REM sleep. Individual detection accuracy in wakefulness was below $80 \%$ in 2 of the participants. The monitor's detection accuracy was less than $80 \%$ in 5 of 10 participants during NREM and REM sleep. Participants with the highest number of detection errors were observed to have more frequent episodes of diminished nasal pressure fluctuations on the simultaneous nasal pressure recording. On review of the false negative detection errors, $94 \%$ could be explained by this phenomenon. The remaining $6 \%$ of detection errors could not be explained after reviewing the nasal pressure signals. That is, a detection error occurred when the participant was observed on the video recording to be wearing the cannula and had clear nasal pressure signals on the simultaneous nasal pressure recording during the monitor's measurement period.

There were no findings on routine history or physical exam that identified those individuals in whom there was lower detection accuracy during wakefulness. Figure 1 shows the relationship between the percentage of correct measurements during sleep and the apnea-hypopnea index in all participants.

The relationship across all participants was not significant $\left(\mathrm{R}^{2}=0.08, \mathrm{p}>0.05\right)$. However, when the two participants (participants 8 and 9) with a relatively low AHI and low percentage of correct measurements during sleep were removed from the analysis, a significant negative correlation was present in the remaining 8 participants $\left(\mathrm{R}^{2}=0.93\right.$, $\mathrm{p}<0.001)$. In contrast to the remaining eight participants, the 2 participants with the unusual results showed a significant amount of relatively shallow respiratory-related pressure fluctuations on the simultaneous nasal pressure recording during sleep, even when apneas and hypopneas were absent.

Approximately half of the monitor's measurements that occurred in association with an apnea or hypopnea resulted in a detection error. Those apneas and hypopneas that did not extend over the entire measurement period often allowed enough time for the monitor to detect at least 2 respiratoryrelated nasal pressure cycles, the minimum number required 
Table 3 Detection accuracy of the monitor during wakefulness and sleep

\begin{tabular}{|c|c|c|c|c|c|c|c|c|c|}
\hline \multirow[t]{2}{*}{ Participant } & \multicolumn{3}{|c|}{ Wakefulness } & \multicolumn{3}{|c|}{ NREM sleep } & \multicolumn{3}{|c|}{ REM sleep } \\
\hline & \# Total & \# Correct & $\%$ Correct & \# Total & \# Correct & $\%$ Correct & \#Total & \# Correct & $\%$ Correct \\
\hline I & 97 & 88 & 90.7 & 94 & 87 & 92.6 & 13 & 10 & 76.9 \\
\hline 2 & 119 & 114 & 95.8 & 65 & 62 & 95.4 & 16 & 15 & 93.8 \\
\hline 3 & 137 & 119 & 86.9 & 58 & 22 & 37.9 & II & 2 & 18.2 \\
\hline 4 & 80 & 65 & 81.3 & 87 & 86 & 98.9 & 14 & 14 & 100 \\
\hline 5 & 90 & 85 & 94.4 & 102 & 81 & 79.4 & 5 & 4 & 80.0 \\
\hline 6 & 165 & 142 & 86.1 & 19 & 18 & 94.7 & 1 & 1 & 100 \\
\hline 7 & 115 & 75 & 65.2 & 39 & I & 2.6 & 24 & 5 & 20.8 \\
\hline 8 & 107 & 83 & 77.6 & 58 & 15 & 25.9 & 9 & 0 & 0 \\
\hline 9 & 89 & 73 & 82.0 & 88 & 65 & 73.9 & 12 & 8 & 66.7 \\
\hline 10 & 119 & 113 & 95.0 & 50 & 47 & 94.0 & 5 & 4 & 80.0 \\
\hline Total & 1118 & 957 & 85.6 & 660 & 484 & 73.3 & 110 & 63 & 57.3 \\
\hline Mean & & & 85.5 & & & 69.5 & & & 63.6 \\
\hline SD & & & 9.0 & & & 34.6 & & & 36.8 \\
\hline
\end{tabular}

Notes: Total \# = total number of samples; \# Correct = number of samples in which the monitor determined the correct conditions (ie, cannula-on and cannula-off); \% Correct = percentage of samples in which the monitor determined the correct conditions.

during a measurement to designate the "cannula-on" condition (Figure 2).

The majority-vote filter was applied to measurements obtained prior to and during the polysomnogram, the latter including both wakefulness and sleep periods due to the frequent transitions between the two states during the polysomnogram recordings. The majority-vote filter improved the monitor's overall detection accuracy, over the entire recording period, to $84.9 \%$ (Table 4 ).

Without the filter, ie, unprocessed data, the mean accuracy across participants was $86.2 \pm 9.8 \%$ prior to the polysomnogram and $75.2 \pm 22.5 \%$ during the polysomnogram. When

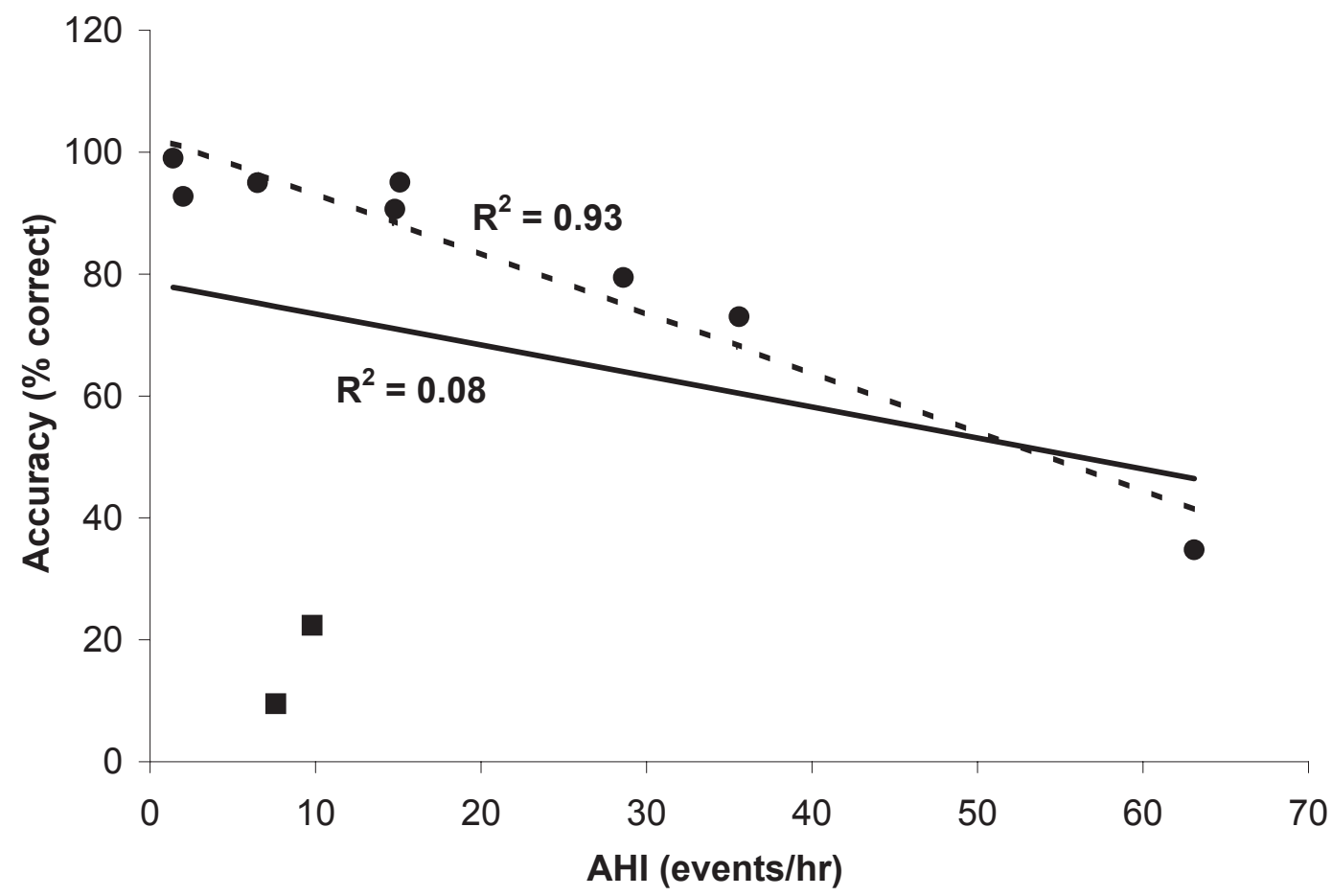

Figure I Percent of correct measurements with the oxygen adherence monitor versus apnea-hypopnea index (AHI) during sleep in all ten participants. The square markers represent the two participants in whom there were a high number of incorrect measurements despite a relatively low $\mathrm{AHI}$. Regression lines are shown for data including all participants (solid line, $R^{2}=0.083$ ) and data excluding two of the subjects (dashed line, excluding square markers, $R^{2}=0.93, p<0.001$ ). 

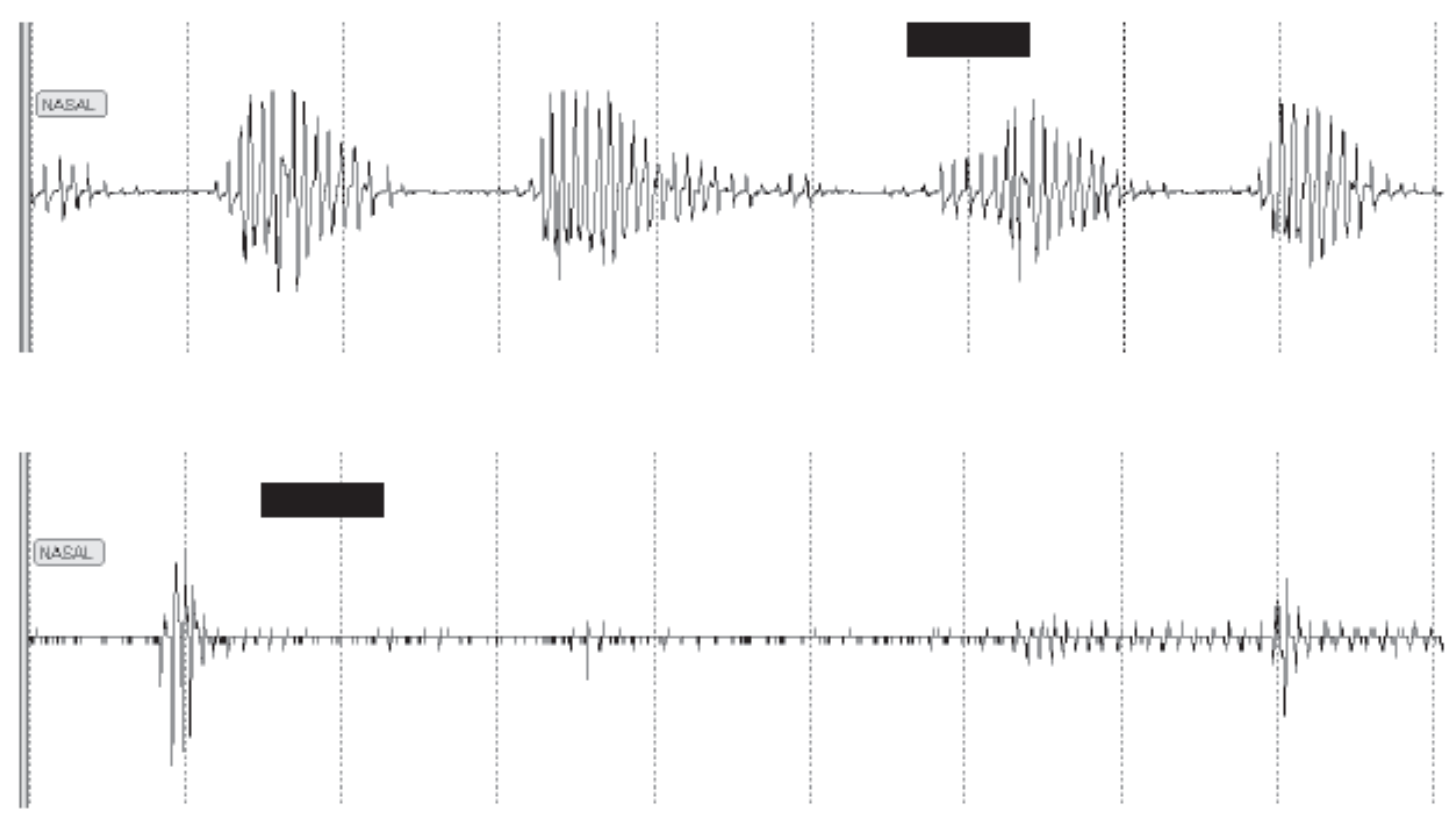

$30 \mathrm{sec}$

Figure 2 Nasal pressure recordings during sleep in two participants who were wearing nasal cannula. The recording in the upper panel reveals repetitive apneas in an individual during NREM sleep.The prolonged period of absent and markedly attenuated nasal pressure signal in the recording in the lower panel occurred in another participant during REM sleep.The solid bars indicate the time that the oxygen adherence monitor obtained one of its 25 second samples. The sample in the upper panel occurred near the end of an apnea; and there were a sufficient number of respiratory-related pressure fluctuations for the monitor to correctly detect that the participant was wearing the cannula. In the lower panel, the pressure fluctuations during the sampling period were of insufficient amplitude; and the monitor incorrectly determined that the participant was not wearing the cannula.

the majority-vote filter was applied to these data, the mean detection accuracy rose to $90.8 \pm 14.4 \%$ prior to the polysomnogram and $81.2 \pm 26.5 \%$ during the polysomnogram. Application of the filter improved detection accuracy in 9 of the 10 participants before the polysomnogram and in 8 of the 10 participants during the polysomnogram.

\section{Discussion}

The results demonstrate the ability of our oxygen adherence monitor to objectively measure whether or not patients with COPD are receiving their oxygen treatment. The monitor's accuracy in detecting whether or not the participants were wearing the nasal cannula, without

Table 4 Effect of applying a majority-vote filter on the percentage of the monitor's correct determinations*

\begin{tabular}{|c|c|c|c|c|c|c|}
\hline \multirow[t]{2}{*}{ Participant } & \multicolumn{3}{|l|}{ Raw data } & \multicolumn{3}{|c|}{ Majority vote filter } \\
\hline & Before PSG & During PSG & Total & Before PSG & During PSG & Total \\
\hline 1 & 89.3 & 91.7 & 90.7 & 96.3 & 99.2 & 98.0 \\
\hline 2 & 94.5 & 96.3 & 95.5 & 96.6 & 99.1 & 98.0 \\
\hline 3 & 91.8 & 57.1 & 69.4 & 100 & 57.3 & 72.3 \\
\hline 4 & 85.7 & 93.6 & 91.2 & 100 & 100 & 100 \\
\hline 5 & 94.8 & 80.8 & 86.3 & 98.7 & 100 & 99.5 \\
\hline 6 & 82.0 & 89.5 & 87 & 91.5 & 100 & 97.2 \\
\hline 7 & 65.2 & 38.6 & 45.5 & 52.3 & 36.2 & 40.2 \\
\hline 8 & 76.3 & 39.4 & 56.3 & 88.5 & 40.2 & 62.4 \\
\hline 9 & 84.9 & 72.4 & 77.2 & 85.9 & 79.8 & 82.2 \\
\hline 10 & 97.1 & 92.4 & 94.3 & 98.5 & 100 & 99.4 \\
\hline Mean & 86.2 & 75.2 & 79.3 & 90.8 & 81.2 & 84.9 \\
\hline SD & 9.8 & 22.5 & I7.| & 14.4 & 26.5 & 20.6 \\
\hline
\end{tabular}

Notes: ${ }^{*}$ Correct determinations $=$ (number of samples correctly identified as cannula-on or cannula-off/total number of samples). 
post-processing, was $79.3 \% \pm 17.1 \%$. Mean detection accuracy was $85.5 \% \pm 9.0 \%$ in wakefulness, $69.5 \pm 34.6 \%$ in NREM sleep, and 63.6\% $\pm 36.8 \%$ in REM sleep. All errors were due to the monitor failing to detect that the participant was actually wearing the cannula. In 8 of the 10 subjects, the percentage of detection errors during sleep appeared to be directly related to the apnea-hypopnea index. The overall detection accuracy of $79.7 \%$ using the raw data improved to $84.9 \%$ with application of a post-processing majority-vote filter.

The results extend our previous study by testing the monitor's performance during different activities of daily living and sleep in patients with COPD (Lin et al 2006). In our previous study, we used direct observation to validate the monitor's performance in patients with COPD. In contrast to the current study, that previous study consisted of a relatively brief protocol, lasting about 1 hour, during periods of sitting and walking that were precisely timed so that the transitions between the different conditions did not occur when the monitor was making a measurement. In addition, the previous study was performed using nasal cannula with a 6-foot length of tubing. Under those circumstances, the monitor had an overall $86 \%$ sensitivity (ie, correctly detecting that the cannula were on) and $100 \%$ specificity (ie correctly detecting that the cannula were off). The current study tested the monitor's performance over a longer period of time during both wakefulness and sleep using 50-ft length nasal cannula, and under less controlled conditions than our previous study (Lin et al 2006). The subjects were free to perform their usual activities of daily living during wakefulness. The results of the current study indicate that the monitor is able to detect the presence or absence of respiratory-related pressure fluctuations at the nares during wakefulness and sleep even when positioned at the end a 50 -ft. length of tubing, the standard length used at home by patients on LTOT.

In the current study, patients were observed simultaneously by three methods: oxygen adherence monitor, video-recording, and continuous nasal pressure recordings. The videorecording served as our "direct observation" to document whether or not the participant was wearing the cannula. These observations allowed us to determine the accuracy of the oxygen adherence monitor. The nasal pressure recordings allowed us to evaluate discrepancies between the oxygen monitor and the video-recording, ie, to differentiate between the technical (ability of the monitor to sense the pressure fluctuations at the nares) and methodological (actual presence of significant nasal pressure fluctuations) causes of the discrepancies.
The technical assessment relates to the monitor's ability to detect respiratory-related pressure fluctuations in the cannula supply line. These small pressure fluctuations from the nares are superimposed on a much larger oxygen supply pressure delivered at the proximal end of a long tube, and then detected through a microprocessor-controlled electropneumatic device. In principle, limitations in the design, construction, and use of the monitor might result in inaccurate detection of breathing. For example, if inspection of the nasal pressure recording showed that there was a good nasal pressure signal, but the monitor did not detect this breathing signal, then we would conclude that there was a technical limitation. A review of the continuous nasal pressure recordings at the time of almost all incorrect measurements showed that the pressure signal during these sampling periods were, in fact, very shallow or virtually flat, indicating that the monitor's inability to correctly detect when the cannula was "on" was due to the method used to assess adherence rather than the monitor's technical performance.

Even if the monitor works perfectly from a technical standpoint, it may still make incorrect observations in subjects who do not breathe normally through the nose. We defined "oxygen use" in terms of the presence of respiratoryrelated pressure fluctuations at the nares. We recognize the possibility that these pressure fluctuations may be absent even though the nasal cannula are placed correctly in the nares. Pharyngeal airway closure during sleep, mouth breathing, and nasal airway obstruction might result in little or no respiratory-related pressure variations at the nares. For example, a shift from nasal to oral breathing might be associated with closure of the retropalatal oropharynx, damping transmission of airway pressure into the nasal passage (Thurnheer et al 2001). Under such circumstances, our device might not sense nasal breathing even when the subject was wearing the cannula and would incorrectly indicate that the subject was not adhering to treatment. This appears to be the explanation for many of the detection errors during sleep. Measurement errors tended to be more common in participants with a higher apnea-hypopnea index, and apneas and hypopneas are associated with absent or relatively low respiratory-related nasal pressure fluctuations. Since the monitor depends on the presence of respiratory-related fluctuations to determine if the patient is using the cannula, the lack of these fluctuations explains why the monitor incorrectly indicated that the cannula was not being worn. Increasing the length of the sample period or the sampling frequency might help reduce the monitor's detection errors in the presence of sleep disordered breathing. 
Six percent of detection errors could not be explained after reviewing the nasal pressure signals. That is, a detection error occurred when the patient was directly observed to be wearing the cannula and the continuous nasal pressure recording showed adequate respiratory-related pressure fluctuations. The most likely explanation is that the continuous nasal pressure recordings during those periods may not have fully represented the signals that were analyzed by the monitor. For example, the oxygen delivery tube could have been slightly compressed during a measurement but the nasal pressure recording tube was undisturbed. The monitor's pneumatic filtering system is sensitive to sharp pressure increases that cause the incoming pressure signal to be out of detection range. In these cases, the monitor detects that the patient is not wearing the cannula. A situation in which this might occur could be when the patient is turning in his sleep and partially resting on the oxygen delivery tube.

In summary, the results further validate the use of a novel monitor to objectively document patient adherence to LTOT. The monitor was most accurate in detecting when participants were not wearing the cannula, yielding $100 \%$ specificity. Excellent results were also obtained when participants were awake and performing daily activities. The most difficult monitoring situation was observed during sleep, and results indicate that accuracy may be reduced in participants who are mouth breathers and/or have frequent apneas and hypopneas. Processing the data with a majority-vote filter improved detection accuracy. The monitor's ability to objectively measure oxygen delivery, rather than oxygen expended, and determine daily patterns of oxygen use will provide valuable information that may lead to new management strategies designed to improve patient adherence to LTOT.

\section{Acknowledgments}

Jerome Beverly, Sharif Branham, and Tracy Seeger provided technical assistance. The authors report no conflicts of interest in this work.

\section{References}

[AASM] American Academy of Sleep Medicine Task Force. 1999. Sleeprelated breathing disorders in adults: Recommendations for syndrome definitions and measurement techniques in clinical research. Sleep, 22:667-89.

[ATS] American Thoracic Society. 1989. Indications and standards for cardiopulmonary sleep studies. Am Rev Respir Dis, 139:559-68.

Avdeev SN, Aisanov ZR, Chuchalin AG. 1999. Compliance as a critical issue in long-term oxgyen therapy. Monaldi Arch Chest Dis, 54:61-6.

Criner GJ. 2000. Effects of long-term oxygen therapy on mortality and morbidity. Respir Care, 45:39-51.

Dunne PJ. 2000. The demographics and economics of long term oxygen therapy. Respir Care, 45:223-8.

Granados A, Escarrabill J, Borras JM, et al. 1997. The importance of process variables analysis in the assessment of long-term oxygen therapy by concentrator. Respir Med, 91:89-93.

Howard P, Waterhouse JC, Billings CG. 1992. Compliance with long-term oxygen therapy by concentrator. Eur Respir J, 5:128-9.

Lin SKV, Bogen DK, Kuna ST. 2005. A novel device to measure patient adherence to long-term oxygen therapy: A validation study. Proc Amer Thoracic Soc, 2:A386.

Lin SKV, Kuna ST, Bogen DK. 2006. A novel device for measuring longterm oxygen therapy adherence: A preliminary validation. Respir Care, 51:266-71.

[MRC] Report of the Medical Research Council working party. 1981. Long term domiciliary oxygen therapy in chronic hypoxic cor pulmonale complicating chronic bronchitis and emphysema. Lancet, 1(8222):681-6.

Nocturnal oxygen therapy trial group. 1980. Continuous or nocturnal oxygen therapy in hypoxemic chronic obstructive lung disease. Ann Intern Med, 93:391-8.

Pierson DJ. 2000. Pathophysiology and clinical effecs of chronic hypoxia. Respir Care, 45:39-51; discussion, p. 3.

Thurnheer R, Xie X, Bloch KE. 2001. Accuracy of nasal cannula pressure recordings for assessment of ventilation during sleep. Am J Respir Crit Care Med, 164:1914-9.

Ting D, Prasada B. 1980. Digital processing techniques for encoding of graphics. Proc IEEE, 68:757-69. 